\title{
Design of Health Management System Based on the Internet of Things
}

\author{
Qian Yao \\ Xi'an Medical University, Xi'an, 710021, China
}

Keywords: Internet of Things; Health management; Design of system

\begin{abstract}
With the development of society and continuous improvement of overall living standard of the society, people increasingly pay more attention to their own health. The traditional medical service system fails to satisfy the increasingly growing needs and existing treatment, prevention and other links have their limitations. To popularize health information and to supply the high-quality resources and services, the design and application of health management system based on the Internet of Things have become the hotspots for researches nowadays. The system builds the individual health management platforms by reasonably using the existing mobile terminals so as to realize that the user can learn his own health situation at any time anywhere and to timely and correctly obtain health service and recommendations. The main studying content of this paper is exactly how to build the design and application of health management system based on the mobile platform.
\end{abstract}

\section{Introduction}

With the rapid development of society, people's living standard is improved ceaselessly; meanwhile, an individual's mental pressure is also increasingly growing. A growing number of people begins to pay attention to their health conditions, raising many new requirements for the traditional medical services. The medical service gradually turns into precaution first. Health management is actually a process to manage the health risks of people and realizes the maximization of individual health condition through building a scientific concept and adjusting the lifestyle. In this phase, the low awareness rate of scientific health knowledge and concept goes against to people's cultivation of a correct lifestyle, so it is especially important to construct a health management system which can supply scientific and correct health information and is convenient for people for shortcut operations. Nowadays, the Internet of Things technology and mobile communication terminals can realize such conception, satisfy people's urgent need for health and, meanwhile, alleviate the pressure for lacking high-quality medical resources in the society.

\section{Basic introduction to the Internet of Things}

The Internet of Things is actually a kind of internet to connect things and is an inevitable development trend for internet and network. The principal and essential feature of the Internet of Things is intelligent service, which is able to install all kinds of sensing devices, which can be rationally connected to form a network to gradually form the internet of things accordingly. The continuous development and application of the internet of things is gradually changing the traditional medical system and ceaselessly building the medical pattern dominated by patients. The specific application fields of the internet of things cover the several items below: supply of medical information, medicine management, blood management, remote management and medical apparatus and instrument management etc. The construction of internet of medical things makes the medical management platform develop in the directions of mobilization and intelligence. The prosperous development of mobile communication technology and devices also propels the innovation and development of application modes of medical service and realizes the validity and timeliness of health management. 


\section{Research situation of the internet of things at home and abroad}

At the World Summit on Information Society held in 2005, the International Telecommunication Union (ITU) published the concept of internet of things and put forward the key technologies of internet of things, features of internet of things and future development directions and trend accordingly. Besides, after the sense of China and the idea of intelligent earth of America were put forward, a growing number of nations begun to study and analyze the internet of things technologies in this phase. At the plenary session of internet of things convened in France in 2008, the future society's challenges and opportunities concerning the internet of things and individual privacy issues etc. during the use of the internet of things were raised. The European technical experts and the European Commission mainly put the research emphasis on the EPC framework, in the hope of establishing a set of reasonable, distributed and fair standards.

In this phase, our country is in a world leading level with respect to the research of internet of things and the internet of things probably will create a mature smart network platform for China. As early as 1999, the Chinese Academy of Science and the principal nations researching the internet of things in the world synchronously studied and analyzed the sensor network and achieved huge developments in micro sensor, mobile base station, wireless intelligent sensor and sensor terminal. Over recent years, such research has gradually become a industry with certain demonstration effect and has obtained large breakthroughs in techniques, materials, system, devices etc. In 2009, Xi'an Youshi Micro-electronics Company developed "No. 1 Tangsic", which became the first Chinese core for the internet of things and is applicable to wireless local area network (WLAN), wireless sensor network, active RFID and other application fields. Besides, the internet of things is gradually applied to the several aspects of military below: smart dust, unattended ground sensor group, smart sensor network, target-located network embedded system technology etc. Besides, for the relatively independent ground radar system and independent satellite, the sensor network based on the internet of things has the following several advantages: firstly, with the features of low cost and highly residual design principle etc., the sensor network is convenient for the realization and construction of the system of internet of things; secondly, the surrounding noises will decrease the overall performance of system. Since the sensor network can realize the remote monitoring, the sensor network can reduce such negative influences to the highest degree; thirdly, the sensor network can successfully realize multi-node union, realizing convenience and feasibility in a large area of monitoring area; fourthly, the sensor network has an excellent self-adaptability of network and the durability and robustness of its application are especially outstanding.

\section{Design principle for health management system based on the internet of things technologies}

The individual health management system based on the internet of things mainly establishes the information bridge among the user, medical organization and service center with the mobile communication devices. The user and doctor can communicate and interact with each other at any time. The user can move the collecting device or sensor for physical health data on the communication terminal to conduct comprehensive analysis and treatment through the health management system and can concentrate and send the user's individual key data every day to the server of central organization for saving, follow-up analysis and diagnosis. The construction of this system not only builds the health data archive for individuals but also provides convenience for the medical staff to develop rapid diagnosis and offer effective and timely services. The main principle of such health management service system is listed in the diagram below. 


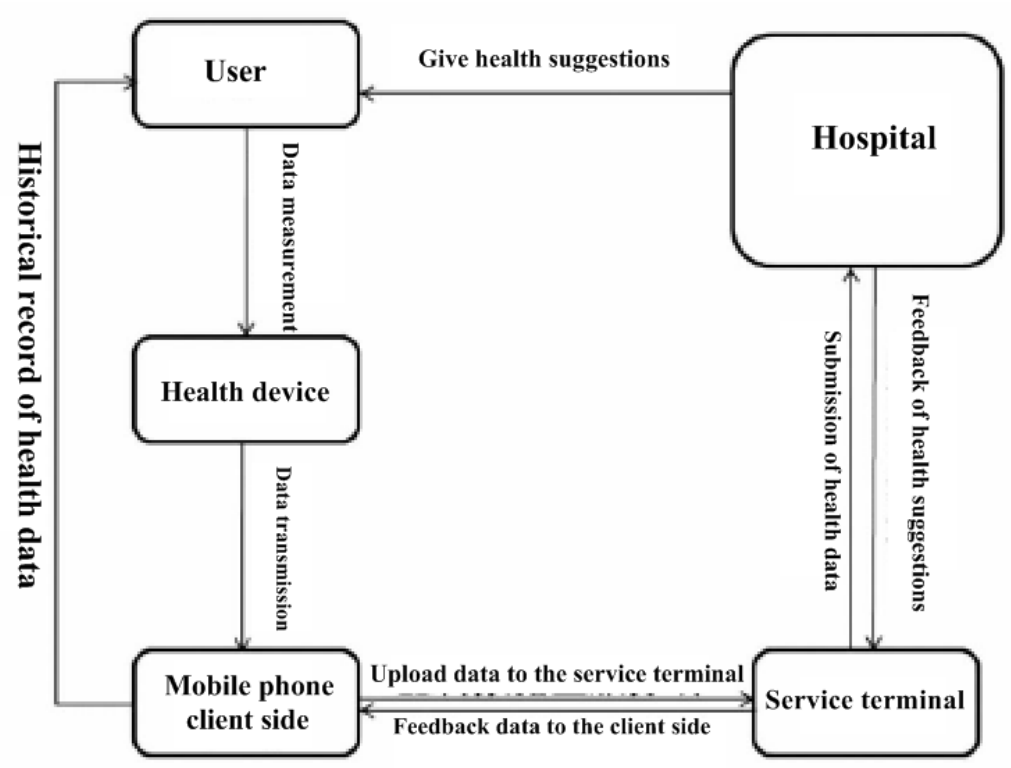

Fig 1. Schematic diagram for the health management system

The basic procedures for such health management system are as follows: firstly, the user monitors and measures all physical data with the devices; the mobile terminal can establish connections to acquire data by using the wireless data transmission (such as Bluetooth). Secondly, the server can receive and save the data for individual health system for comprehensive analysis. Thirdly, the medical staff of the hospital can visit the user's individual health data under authorization accordingly and give corresponding medicine guidance and treatment suggestions and feed back to the individual customer side directly through the system. The user can also learn his own health condition at any time to timely adjust and improve his lifestyle via the historical data and record. Gradually build the correct health concept under the long-term use of health management system and close recommendation alert service. The healthy lifestyle will finally realize the optimization of individual health resources and the maximum utilization of social medical resources.

\section{Module design based on the health management system of internet of things}

There is a vast majority of users to operate by using the android mobile operating system with open source codes; move the terminal CS pattern to construct the overall structure of system; design the android customer side in the MVC pattern; reasonably use the Web Service techniques to fully realize the data communications between the server and customer side; image the server and client side data by using the visualization technology.

\section{Account management}

Once the user's individual account is registered, the system will establish a corresponding individual data archive for him. Apart from the user name and password information, the registration will also complete the basic information of an individual through questionnaires, such as real name, age, gender, address, past medical history, emergency telephone number and other detailed information, so as to provide the follow-up medical staff with reliable references for diagnosis. Not only the user but also the authorized medical staff can visit the account information at any time. When logging in, the user can enter into the system interface fast by only offering user name and password.

\section{Data acquisition module}

The Android system of high version can support Bluetooth communication, can form a connection between a smart phone and equipment via the SPP agreement transmission function of Bluetooth and can carry out actual communications via the serial port after successful connection. One can rapidly realize the data transmission between the health monitoring device and the mobile communication terminal via the Bluetooth technology. The collection of individual physical data is the premise of 
comprehensive analysis by the health management system; both the accuracy and reliability of data will influence the result of analysis and the quality of diognosis service.

\section{Data image and visualization module}

The fundamental philosophy of design system visualization is as follows: among mass data, divide each data into a image unit and the collection of many data forms the corresponding data image; show the properties of different data with the multidimensional data modules; change the angle of data observation for further research and analysis. The data visualization has the three features of interaction, visualization and multidimensional nature. Firstly, interaction. The interaction is actually to use the operability between the user and computer and the relevant techniques for data visualization to guarantee that the user can manage and develop data information through interaction. Secondly, multidimensional nature. The multidimensional nature is actually that the object or time may have multiple properties or variations, which can be classified, combined and sequenced by data according to varied dimensions by using the data visualization technologies. Thirdly, visualization. Visualization actually is to show the data information through curves, images, pictures, 3D. animation and 2D pictures to carry out visualization analysis on the interrelationships and display mode among data.

\section{Data uploading module}

Web Service technology can use different applications to be applied in a machine without attached or a third-party hardware or software so as to achieve the goal of mutual interchange of data. Reasonably standardize the relationship among all applications with the Web Service technology and corresponding exchanges must be conducted regardless of the platforms, languages and agreements. The Web Service technology mainly is the self-contained and self-described network modules and is actually a relatively regular technology, such as HTTP and XML. The Web Service technology can effectively cut the cost for system interfaces and can also offer a reasonable communication platform and mechanism among enterprises and multiple organizations. The JSON can be used to realize such function.

\section{Key techniques for health management system}

The Android platform which is composed of middleware, operating system, applications and user interface can open the source code to the public and, meanwhile, is also the first mobile platform only open to the mobile terminal travel. The Android system can reasonably put the hardware on different platforms via the original codes. Since the Android system has the features of complete opening and easy design, the system has become the most important operating system for mobile phone, including the following four aspects:

\section{Application layer}

In the application layer, the Android system mainly covers a series of communication establishments, which are mainly SMS program, email client side and contacts etc. That is to say, basically most of the applications are designed based on the Java language. Therefore, it is extremely important to use Java language during the design of application layer.

\section{Application framework}

The programming personnel, which desires to reasonably use the API in the program framework, can use the corresponding core programs for direct invoking. Besides, such operating mode exists in the application framework. Therefore, reasonably design the frame layer as the simple and convenient reuse establishment. During the development of Android application, it is necessary to operate relatively bottomed applications. So to speak, all the applications in the Android system are relatively equal and both the commonly developed applications and those developed by the Android system can visit the API offered by the Android system. 


\section{Library function}

In the actual design process, the Android system covers many $\mathrm{C}++$ and $\mathrm{C}$ function libraries, different components and different databases, so it is very convenient to exchange of use between the Android system. Generally speaking, it is not allowed to call $\mathrm{C}++$ or $\mathrm{C}$ function libraries in developing the Android system but it is feasible to reasonably use the application frame of Android system to offer the function libraries needed by design.

\section{Linux kernel}

Actually, the Android system is designed on the basis of Linux and the design of Android system shall be established on the basis of Linux2.6, which is an enhanced edition actually. In this phase, the Android's Linux kernel offers memory management, networking protocol, safety, driving model and other main service systems for the design of system. Besides, Linux kernel is also the gradual cascading of software and hardware.

\section{Conclusion}

The continuously developing and expanding internet of things technologies it will certainly become today's research hotspots and trend to build the structure and application of a mobile individual health management system by using the mobile communication technologies and data analysis platform and by combining the development conditions for internet and medical treatment.

\section{Acknowledgments}

This paper is a part of special fund program of Shaanxi Education Department, Program NO.:2013JK1153.

\section{References}

[1] Wang Yu, Xu Yuanhong and Yanghong etc. Application Framework of the Internet of Things Technology in Patients’ Health Management. Chinese Hospital, 2010,14(8):1-4.

[2] Fan Chun and Yu Guangjun. Remote Health Management Based on the Internet of Things Technology. China Digital Medical Science, ,2011,06(1):58-60.

[3] Zhou Guangqing, Wu Shuyu and Xue Bingbing etc. Design and Realization of Community Health Management Platforms Based on the Internet of Things. Medical and Sanitary Equipment, 2014,35(8):53-56,101.

[4] Sun Yao, Li Wenyuan and Ai Yuhua etc. Construction and Research on Self-health Management Mode of the Old-age Chronic Illness Based on the Internet of Things. Chinese Journal of General Practice, 2014(10):1164-1166,1171.

[5] Zhang Dongni. Investigation on Acceptability and Influence Factors of the Elderly Home Health Management System Based on Technology Acceptance Model Theories. Nanjing Medical University, 2013:223. 